Document downloaded from:

http://hdl.handle.net/10251/127475

This paper must be cited as:

Perez, J.; Morant, M.; Llorente, R.; Martí Sendra, J. (2009). Joint Distribution of PolarizationMultiplexed UWB and WiMAX Radio in PON. Journal of Lightwave Technology.

27(12):1912-1919. https://doi.org/10.1109/JLT.2009.2022342

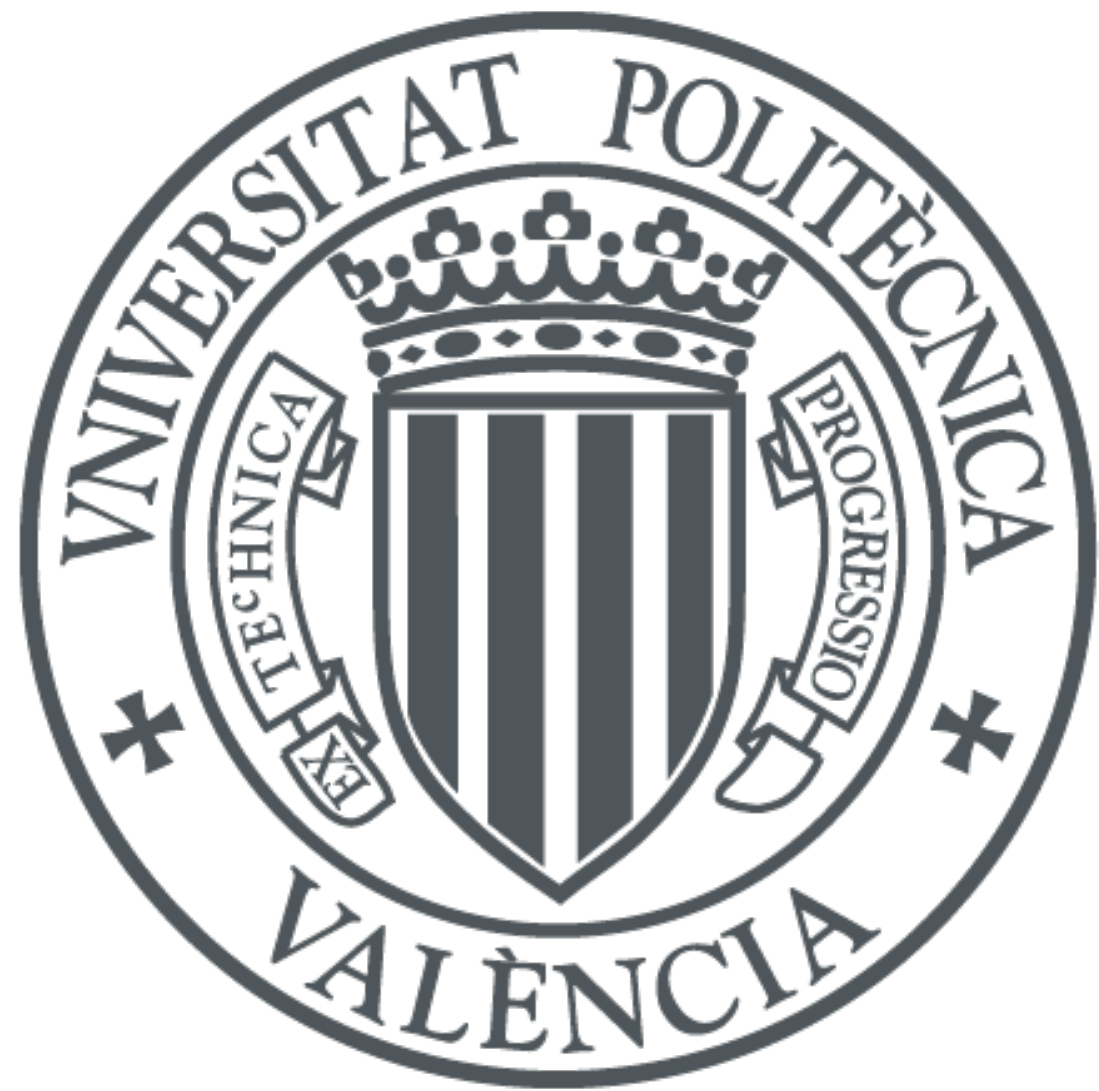

The final publication is available at

http://doi.org/10.1109/JLT.2009.2022342

Copyright Institute of Electrical and Electronics Engineers

Additional Information 


\title{
Joint Distribution of Polarization-Multiplexed UWB and WiMAX Radio in PON
}

\author{
Joaquin Perez, Maria Morant, Roberto Llorente and Javier Marti
}

\begin{abstract}
In this paper, the feasibility of the joint distribution of ultra-wideband (UWB) and WIMAX wireless using polarization multiplexing as a coexistence technique is proposed and experimentally demonstrated within the framework of passive optical networks (PON). Four single- and orthogonal-polarization multiplexing schemes are studied targeting to reduce the mutual interference when UWB and WiMAX are distributed jointly through standard single-mode fiber (SSMF) without transmission impairments compensation techniques and amplification. Experimental results indicate successful transmission up to $25 \mathrm{~km}$, in SSMF exceeding the range in typical PON deployments. The radio link penalty introduced by optical transmission is also investigated in this paper.
\end{abstract}

Index Terms- Optical fiber communication, radio-over-fiber, polarization division multiplexing, integrated optical-wireless access, ultra-wideband radio, WiMAX radio

\section{INTRODUCTION}

$\mathrm{P}$ assive optical networks (PON) are a fiber-to-the-home (FTTH) access technology of special interest nowadays under deployment around the world [1]. PON access the customer premises employing repeater-less optical power splitting and standard single-mode fiber (SSMF). PON technology is preferred when areas with a larger number of users must be served [2]. PON leads to a more economical network deployment than other point-to-point optical access technologies due to several factors: (i) Transmission impairments, such as group-velocity dispersion (GVD) or polarization-mode dispersion (PMD), are not required to be compensated in most cases [3]. (ii) User aggregation is done by passive splicing new fibers, giving more flexibility and scalability than optical access networks with in-line amplification, which would require careful network planning [4]. (iii)Fiber breaks can be easily repaired, reducing maintenance costs. Nevertheless, the straightforward

Manuscript received December 1, 2008. This work has been partly funded by the European Commission through the FP7 ICT 216785 UCELLS project [23] in the 7th ICT-Framework Programme [24][25]. Maria Morant's work is supported by Spain FPU MICINN grant AP2007-01413.

Joaquin Pérez, Maria Morant, Roberto Llorente, and Javier Marti are with the Networks and Systems Area of the Valencia Nanophotonics Technology Centre (www.ntc.upv.es), Universidad Politécnica de Valencia, Spain. E-mail: \{joapeso, mmorant, rllorent, jmarti\} @ntc.upv.es.

Copyright (c) 2009 IEEE. Personal use of this material is permitted. However, permission to use this material for any other purposes must be obtained from the IEEE by sending a request to pubs-permissions@ieee.org implementation in PON leads to a reach limitation in the optical transmission since amplification, regeneration and impairment compensation stages are eliminated along the optical link. Typical PON reach distances have been reported to be around $20 \mathrm{~km} \mathrm{[5].}$

The distribution of wireless standards in optical access networks, known as hybrid fiber-radio access [6], is an interesting approach that exhibits several advantages: (i) Optical access networks are capable to distribute wireless radio at frequencies above $60 \mathrm{GHz}$ if external modulation is employed [7]. (ii) No trans-modulation is required at customer premises since the wireless signal is transmitted through the optical path in its native format. (iii) No frequency upconversion is required at customer premises. The wireless signal is photodetected, filtered, amplified and radiated in order to establish the wireless connection. (iv) Optical access networks are transparent to the specific modulation employed. This flexibility is of special interest for operators as wireless standards and regulation evolve at fast rate.

Regarding current wireless standards, UWB radio transmission technology has been proved to be adequate for the distribution of uncompressed high definition audio/video in hybrid fiber-radio networks [8]. UWB targets short-range high-bitrate communications, potentially exceeding $1 \mathrm{Gbit} / \mathrm{s}$ [9]. Moreover, UWB is receiving increasing interest because its low self-interference, tolerance to multi-path fading and potential low-cost characteristics [10]. In the near future it is expected the pervasive presence of UWB transmitters supporting a broad range of applications, from wireless computer universal serial bus (WUSB) to home multimedia wireless communication systems, such as wireless high definition multimedia interface (HDMI) [11]. UWB is defined as a radio modulation technique with $500 \mathrm{MHz}$ of minimum bandwidth or at least $20 \%$ greater than the center frequency of operation [12]. The modulated signal is required to fulfill stringent equivalent isotropic radiated power (EIRP) limits. Two specific UWB implementations are mainstream nowadays: impulse-radio (IR-UWB), which transmits data by short impulses (monopulses), and orthogonal frequency division multiplexing (OFDM-UWB), which divides the UWB spectrum into 14 channels of $528 \mathrm{MHz}$ bandwidth (BW). In this last case, each channel is occupied by one OFDM signal composed by 128 carriers, and each carrier can be QPSK- or DCM-modulated [13].

WiMAX, worldwide interoperability for microwave access, is a wireless transmission technology targeting medium- to 
long-range data communications at bitrates up to $12 \mathrm{Mbit} / \mathrm{s}$ [14]. WiMAX is expected to replace large wireless local-area network installations [15], e.g., University campus, commercial areas, etc. Comparing the bitrate and expected range, WiMAX and UWB are complementary radio technologies expected to coexist in a near future.

The transmission of UWB radio for audio/video distribution was first proposed for FTTH networks with optical amplifier sections in [8], where a single wavelength signal without polarization multiplexing is distributed per user. Since UWB can be regarded as a low cost technology, a reduction of the overall network deployment costs is expected. Another advantage arises from the use OFDM-UWB as defined in WiMedia UWB [13], which is especially well suited for uncompensated and un-amplified PON. Nevertheless, OFDMUWB and QAM-WiMAX modulation formats require higher linearity on the radio over fiber (RoF) distribution system [16] than less complex modulations, such as on-off keying (OOK) modulation. UWB and WiMAX coexistence on low-cost multi-mode fiber (MMF) was reported in [17] for indoor applications. In this case, a single wavelength signal without any polarization multiplexing technique was employed.

To the best of our knowledge, this paper proposes by the first time the joint distribution of UWB and WiMAX radio over SSMF employing a polarization-multiplexing scheme. Different single- and orthogonal-polarization schemes are proposed and analyzed aiming to minimize the UWB and WiMAX mutual interference when distributed through SSMF and no amplification or transmission impairment compensation techniques are employed. The experimental results indicate successful transmission up to $25 \mathrm{~km}$ in SSMF, which exceeds the range in typical PON deployments [5]. Moreover, the impact of the optical transmission in the radio path for this PON distribution system is also reported in this paper.

This paper is structured in five sections: Section II describes the polarization multiplexing joint distribution of UWB and WiMAX concept. Section III presents the experimental results of UWB transmission on a single polarization wavelength. This is the baseline performance for further comparison. Section IV describes the polarization multiplexing technique proposed. Three polarization multiplexing schemes are considered in the experiments and their performance on the joint UWB and WiMAX RoF distribution measured. Finally, the main conclusions are drawn in Section V.

\section{UWB AND WIMAX RADIO DISTRIBUTION IN PON}

The proposed technique for joint UWB and WiMAX radio distribution in PON is depicted in Figure 1.

Figure 1 shows a central node (central office, $\mathrm{CO}$ ), which can generate standard UWB and WiMAX wireless signals, indicated as generic Wireless 1 and Wireless 2 blocks in the figure. These radio signals are typically converted to the optical domain by external modulation. Both optical signals, after polarization adjustment, are combined by a polarization beam splitter/combiner (PBS) and distributed through a SSMF-based optical access network to a given number of subscribers. At the subscriber premises, the optical signal is adjusted in polarization to match the slow and fast axis of a reception PBS in order to separate the two orthogonal polarizations. Once each polarization is splitted, the individual wireless signals are photodetected, filtered, amplified and directly radiated to the users present at the customer premises. This approach benefits from the high bitrate capabilities of UWB, supporting bitrates up to $1 \mathrm{Gbit} / \mathrm{s}$ at a few meters range [9], which can be extended to $30 \mathrm{~m}$ by multiple-input multiple-output (MIMO) processing [18]. Furthermore, the WiMAX radio complements UWB providing coverage to the whole home/building at a lower bitrate of $2 \mathrm{Mbit} / \mathrm{s}$ [14][19].

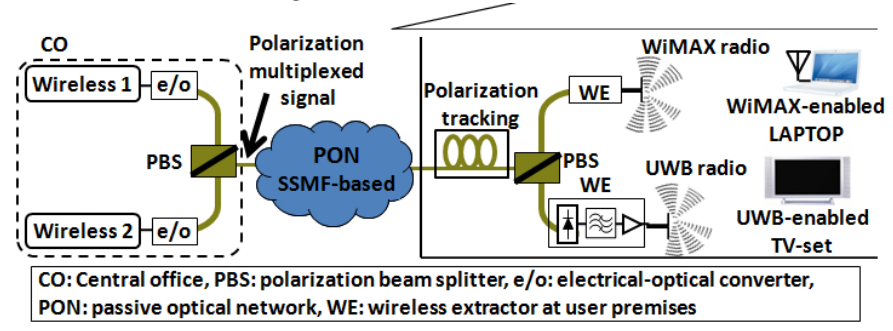

Figure 1. Concept of joint wireless services distribution by polarization multiplexing in hybrid fiber-radio access on PON.

The approach depicted in Figure 1 permits a high spectral efficiency. Two polarizations are distributed per user, each one carrying UWB or WiMAX radio. Multi-user operation can be implemented by wavelength-division multiplexing (WDM) and/or sub-carrier multiplexing (SCM) techniques [20]. In our system, simultaneous UWB and WiMAX transmission in a single channel is analyzed for demonstration purposes. Both transmitted UWB and WiMAX signals are based on OFDM modulation with a spectral efficiency of $0.3788 \mathrm{bit} / \mathrm{s} / \mathrm{Hz}$ and $0.634 \mathrm{bit} / \mathrm{s} / \mathrm{Hz}$, respectively. As shown in Section IV, this spectral efficiency is almost doubled when the polarization multiplexing technique is introduced.

\section{SINGLE POLARIZATION UWB DISTRIBUTION}

Figure 2 shows the experimental setup used to evaluate the performance of UWB radio distribution in PON SSMF path distances. The transmitted UWB signal follows the WiMedia OFDM-UWB standard [13]. This signal comprises two channels (generated by a Wisair DV9110 module) with $528 \mathrm{MHz}$ bandwidth. Each channel bears one OFDM signal comprised by 128 QPSK-modulated carriers, 6 null carriers, and 12 pilot tones. The channel bitrate is $200 \mathrm{Mbit} / \mathrm{s}$, providing an aggregated bitrate of $400 \mathrm{Mbit} / \mathrm{s}$ per user. Each channel is centered at $3.432 \mathrm{GHz}(\mathrm{Ch} \mathrm{1)}$ and $3.96 \mathrm{GHz}$ (Ch 2), respectively (Figure 3 ). The two UWB channels are modulated on a Mach-Zehnder electro-optical modulator (MZ-EOM in Figure $2, \mathrm{~V}_{\pi}=4.5 \mathrm{~V}$ ) and are boosted by an optical erbium doped fiber amplifier (EDFA in Figure 2, Amonics 30-B-FA) at the $\mathrm{CO}$ output and transmitted through 5,10 and $25 \mathrm{~km}$ SSMF paths. These lengths correspond to the expected distances in PON access. The PMD effect associated to these SSMF lengths is negligible since the SSMF fiber used in this experiment shows a very-low PMD factor (first-order approximation) of $0.08 \mathrm{ps} / \mathrm{km}^{1 / 2}$. Other factors such as GVD 
and chromatic dispersion, reflected in the carrier suppression effect [21], are not limiting factors for the optical transmission distances under consideration. The OFDM-UWB signals are photodetected and amplified adjusting the EIRP to the $-41.3 \mathrm{dBm} / \mathrm{MHz}$ level allowed in UWB regulation [12].

The error vector magnitude (EVM) is a figure of merit for assessing the quality of digitally modulated communication signals. EVM measurements have been performed on a digital signal analyzer (Agilent DSA 80000B) to evaluate the link degradation experienced by wireless services, in this case UWB, distribution over the system.

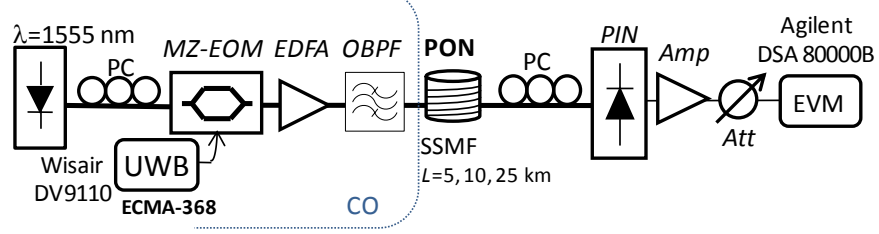

Figure 2. Experimental setup for the performance analysis of UWB radio distribution on a single wavelength on SSMF at PON distances.

Figure 3(b) shows the power spectrum density (PSD) of the two UWB channels where the degradation introduced by $25 \mathrm{~km}$ of optical transmission SSMF can be observed.
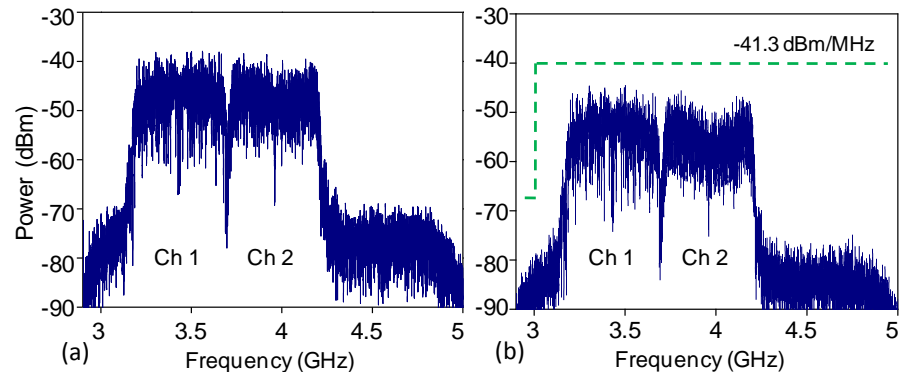

Figure 3. PSD of the UWB spectrum distributed in single polarization, $\mathrm{RBW}=1 \mathrm{MHz}$. (a) Before optical modulation. (b) After $25 \mathrm{~km}$ SSMF transmission, photodetection and amplification. The spectral UWB transmission PSD mask in current regulation [13] is depicted as a dashed line.

It is important to point out that the received power meets in all the cases the PSD limit of $-41.3 \mathrm{dBm} / \mathrm{MHz}$, and the spectral mask in FCC regulation [12]. This guarantees that the received signal at costumer premises could be radiated just photodetecting, filtering and amplifying. The increase in the optical power at fiber distribution can be regulated using a variable attenuator, as shown in Figure 2.

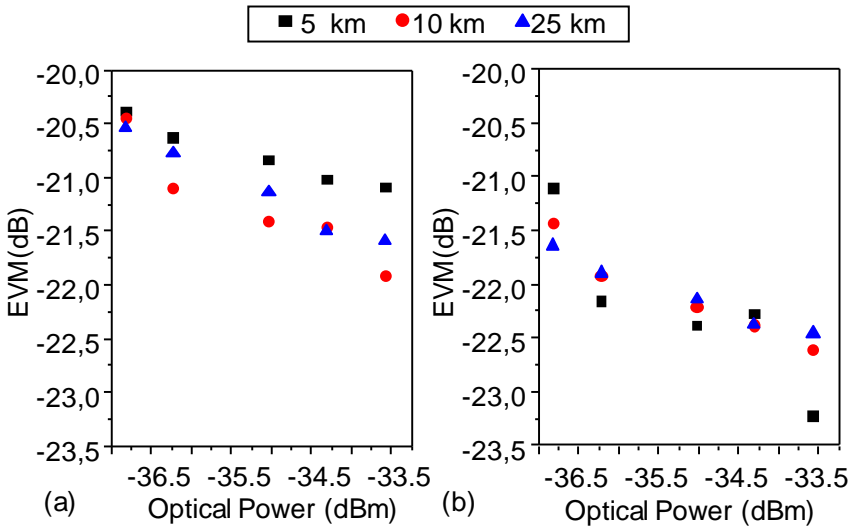

Figure 4. UWB EVM vs. fiber length transmission vs. optical power before photodetection for (a) channel 1 and (b) channel 2.
Figure 4 shows the measured EVM for UWB Ch 1 and Ch 2 channels at different optical power levels measured at the PIN photodetector shown in Figure 2.

The EVM results shown in Figure 4 indicate a soft EVM variation when the received optical power varies in the range under consideration. This UWB EVM variation ranges from -20.75 to $-22 \mathrm{~dB}$ EVM for $\mathrm{Ch} 1$, and from -21 to and $-23 \mathrm{~dB}$ EVM for Ch 2. The EVM results indicate the successful UWB transmission in the SSMF paths from 5 to $25 \mathrm{~km}$, as EVM values are clearly below the $-14.5 \mathrm{~dB}$ EVM UWB threshold defined in [13].

\section{A. Radio transmission penalty}

The impact of UWB distribution in SSMF on the radio path is evaluated in this section. This study aims to ensure that the fiber transmission at PON distances does not strongly affect the radio transmission. In this way, the received UWB signal could be radiated without special transmission impairments compensation algorithms in the OFDM modulation. The performance is studied measuring the UWB EVM at radio distances from 0 to 3 meters after optical transmission through SSMF spans of 5,10 and $25 \mathrm{~km}$.

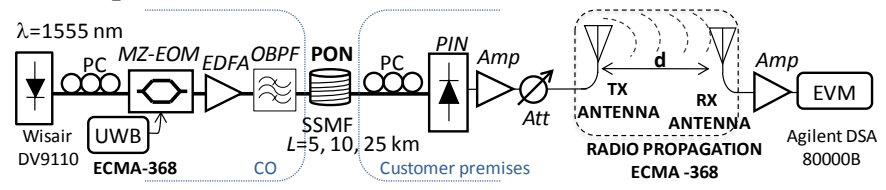

Figure 5. Experimental setup for the analysis of UWB radio penalty introduced after 5 to $25 \mathrm{~km} \mathrm{SSMF}$ optical transmission.

Figure 5 shows the experimental setup employed in this case including the radio transmission path previously discussed. After SSMF transmission, the level of OFDM-UWB signals is adjusted to the PSD regulated level of $-41.3 \mathrm{dBm} / \mathrm{MHz}$. Then, the UWB signal is photodetected, filtered, amplified and radiated by a $0 \mathrm{dBi}$ gain omnidirectional antenna. This signal is detected with a $0 \mathrm{dBi}$ antenna from the same model/manufacturer and its EVM is measured.

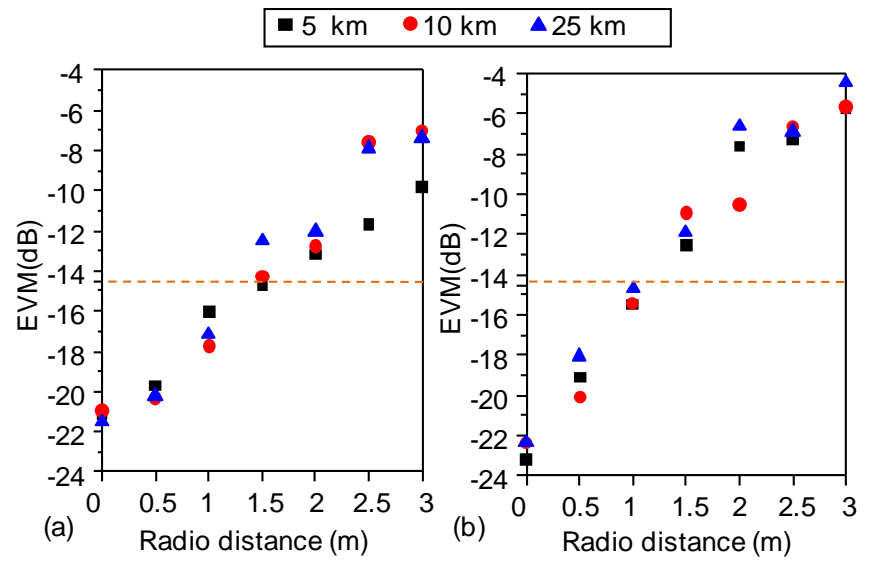

Figure 6. UWB EVM vs. fiber length vs. wireless range for $-34.305 \mathrm{dBm}$ received optical power for (a) channel 1 and (b) channel 2.

The EVM results are shown in Figure 6. The EVM threshold limits the radio distance range after SSMF transmission to $1.5 \mathrm{~m}$ for Ch 1 , after up to $10 \mathrm{~km} \mathrm{SSMF} \mathrm{fiber}$ propagation, or to $1 \mathrm{~m}$ for all fiber lengths. For Ch 2, the EVM 
threshold limits radio distance to $1 \mathrm{~m}$ in all SSMF paths. These distances are in line with the UWB performance, and hence, SSMF transmission does not strongly affect the radio range.

\section{POLARIZATION MULTIPLEXING JOINT DISTRIBUTION}

Three different polarization strategies are analyzed for the joint distribution of UWB and WiMAX radio in this section. These strategies are depicted in Figure 7(b-d): Figure 7(b) shows the co-channel polarization multiplexing strategy where the UWB signal is allocated in the first and second UWB channels [13], marked as $\mathrm{Ch} 1$ and $\mathrm{Ch} 2$, and the WiMAX signal is distributed in the Pol. B shown in Figure 7(a) at the centre frequency of $3.5 \mathrm{GHz}$ [22]. This is the most restrictive situation from the optical transmission point of view since UWB and WiMAX overlap in frequency but are transmitted in different polarizations. Three WiMAX bandwidths are considered (5, 10 and $20 \mathrm{MHz})$ in this case. The second strategy is shown in Figure 7(c): Adjacent-channel polarization multiplexing. This approach is based on transmitting the UWB signal in the second and third UWB channels (Ch 2 and $\mathrm{Ch} 3$ ). In this way, WiMAX and UWB mutual interference is reduced. The third case considered is when UWB radio is transmitted in both polarizations providing the maximum transmission bitrate of $800 \mathrm{Mbit} / \mathrm{s}$ per user. This approach is depicted in Figure 7(d).
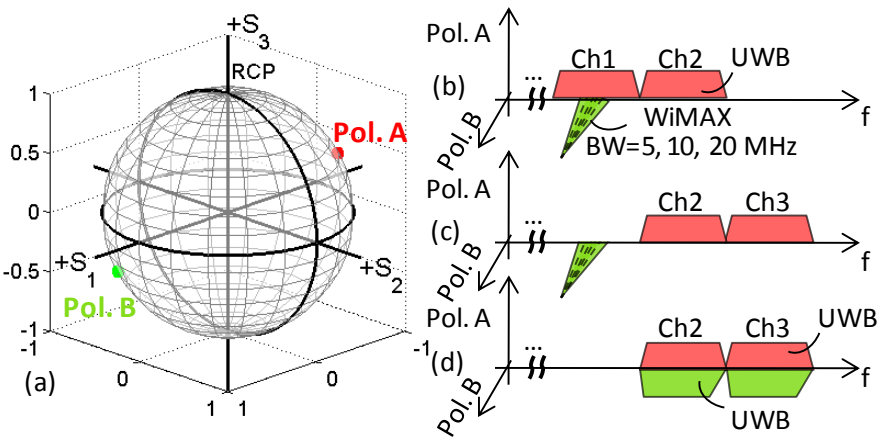

Figure 7. (a) Orthogonal polarizations (measured) launched in the SSMF. (b) Co-channel polarization scheme. (c) Adjacent-channel polarization scheme. (d) UWB polarization multiplexing scheme.

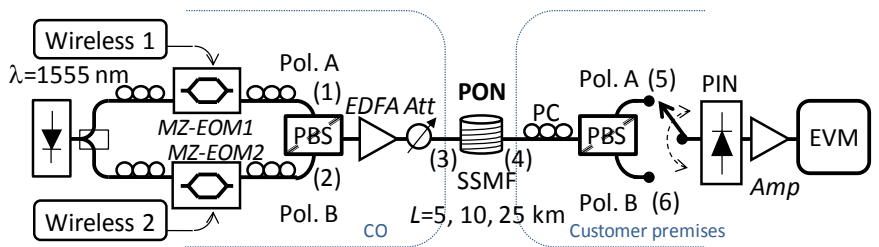

Figure 8. Evaluation setup for optical polarization multiplexing distribution over PON links.

Figure 8 shows the experimental setup employed for the polarization multiplexing transmission. Two different wireless services can be transmitted using the polarization diversity described in Figure 7(a). The two generated signals, UWB or WiMAX, are modulated by a MZ-EOM at quadrature bias (QB) point. The UWB- and WiMAX-modulated optical signal at points (1) and (2) in Figure 8, is adjusted with a polarization controller to linear-horizontal ( $\mathrm{LH}$, named Pol. $\mathrm{A}$ in Figure 7(a)) and linear-vertical states (LV, named Pol. B in
Figure 7(a)), and combined by a PBS to generate the polarization-multiplexed (PM) signal. The PM signal is boosted by and EDFA (Amonics 30-B-FA) at the CO output and launched through SSMF ( $\mathrm{L}=5,10$, and $25 \mathrm{~km})$. The EDFA output level remains constant to not improve the noise introduced by EDFA. However, the total optical power level in point (3) in Figure 8 (optical launch power over PON) is adjusted with a variable attenuator from -3 to $7 \mathrm{dBm}$, to investigate the PM performance from the customer point-of-view.

The PM signal is received at point (4) in Figure 8, where the two polarizations are splitted, photodetected, filtered, amplified and should be radiated to the final user. No demodulation or up-conversion stages are required with this technique. At the receiver, the state of polarization is manually adjusted by a polarization controller (PC). This controller would be an automatic polarizer stabilizer in an on-the-field deployment [23]. After the PC, a PBS resolves polarization A and B (Pol.A, Pol. B) at points (5) and (6) respectively. Each PM polarization signal is detected with a PIN photodetector $(0.7 \mathrm{~A} / \mathrm{W}$ responsivity), amplified and analyzed in order to evaluate the EVM of each signal.

\section{A. Co-channel polarization multiplexing}

In this section, UWB and WiMAX distribution over a PON fiber deployment has been studied. The first case under study is in-band coexistence, where WiMAX and UWB signals coexist on the PON fiber deployment but they are transmitted over an orthogonal polarization in order to minimize mutual interference between them. The two orthogonal polarizations shown in Figure 7(a) are employed in this case. The setup shown in Figure 8 is used to analyze the co-channel polarization multiplexing scheme. Wireless service 1 (in Figure 8) is a UWB signal that uses sub-band \#1 and subband \#2 (center frequencies of $3.432 \mathrm{GHz}$ (Ch 1) and $3.96 \mathrm{GHz}$ (Ch 2), respectively) and service 2 is a WiMAX signal operating in the $3.5 \mathrm{GHz}$ band.

The setup for wireless service 1 comprises UWB multiband-OFDM (MB-OFDM) signal generation by Wisair DV-9110 modules as described in the previous section. The UWB signal is generated following the WiMedia-defined UWB specification described in the ECMA-368 standard [13]. The wireless service 2 is a WiMAX signal that corresponds to a broadband wireless access (BWA) indoor terminal following IEEE 802.16e standard [14]. WiMAX utilizes scalable orthogonal frequency division multiple access (SOFDMA) QPSK modulation. The signal is centered at $3.5 \mathrm{GHz}$ following the European regulation [22]. The main WiMAX signal parameters are summarized in Table I.

TABLE I. WIMAX SIGNAL PARAMETERS

\begin{tabular}{|l|c|c|c|}
\hline Center frequency & \multicolumn{3}{|c|}{$3.5 \mathrm{GHz}$} \\
\hline Bandwidth & $5 \mathrm{MHz}$ & $10 \mathrm{MHz}$ & $20 \mathrm{MHz}$ \\
\hline FFT-points & \multicolumn{3}{|c|}{1024} \\
\hline Subchannel spacing & $5.46875 \mathrm{kHz}$ & $10.9375 \mathrm{kHz}$ & $21.875 \mathrm{kHz}$ \\
\hline Oversampling rate & \multicolumn{3}{|c|}{$1 / 28$} \\
\hline Guard period & \multicolumn{3}{|c|}{ QPSK $(1 / 2 \mathrm{CTC})$} \\
\hline Symbol duration & $182.86 \mu \mathrm{s}$ & $102.85 \mathrm{~s}$ & $57.14 \mu \mathrm{s}$ \\
\hline Modulation & \multicolumn{4}{|c|}{$6.34 \mathrm{Mbit} / \mathrm{s}$} & $12.68 \mathrm{Mbit} / \mathrm{s}$ \\
\hline Downlink Data rate & $3.17 \mathrm{Mbit} / \mathrm{s}$ & \multicolumn{3}{|c|}{}
\end{tabular}


The WiMAX signal is synthesized by software (Agilent N7615B signal studio) and generated by a vector signal generator (Agilent ESG 4483C). Three possible bandwidths (5, 10, and $20 \mathrm{MHz})$ are considered in the transmission performance measurements.
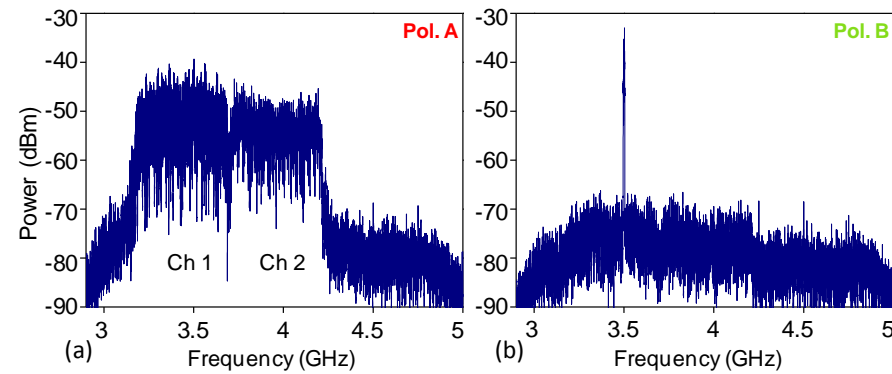

Figure 9. RF received spectrum after $25 \mathrm{~km}$ SSMF transmission measured with in-band coexistence in (a) point (5) Pol. A, and (b) point (6) of Figure 8 Pol. B. RBW = $1 \mathrm{MHz}$.

Figure 9 shows RF spectrum after $25 \mathrm{~km}$ SSMF transmission at point (5) and (6) of the setup depicted in Figure 8. It should be noticed that rejection ratio between Pol. B and Pol. A of the PBS is around $30 \mathrm{~dB}$. Figure 9(a) is the RF spectrum with Pol. A that comprises a $400 \mathrm{Mbit} / \mathrm{s}$ aggregated MB-OFDM UWB signal. Figure 9(b) shows the WiMAX $10 \mathrm{MHz}$ bandwidth signal with Pol. B.

In Figure 10, the EVM measured for each wireless service (UWB, WiMAX) and for each polarization (Pol. A, Pol. B) in the co-channel polarization multiplexing configuration is depicted. EVM results for UWB signal shows that at $25 \mathrm{~km}$ PON transmission, the UWB EVM threshold of $-14.5 \mathrm{~dB}$ is always accomplished [13]. Therefore, as optical launch power over fiber increases, the UWB transmission quality is improved. The EVM results shown in Figure 10(b) indicates that WiMAX distribution at $25 \mathrm{~km}$ PON distance is also feasible as the EVM $-15 \mathrm{~dB}$ limit recommended in the standards [14] is always fulfilled in the experimental work.

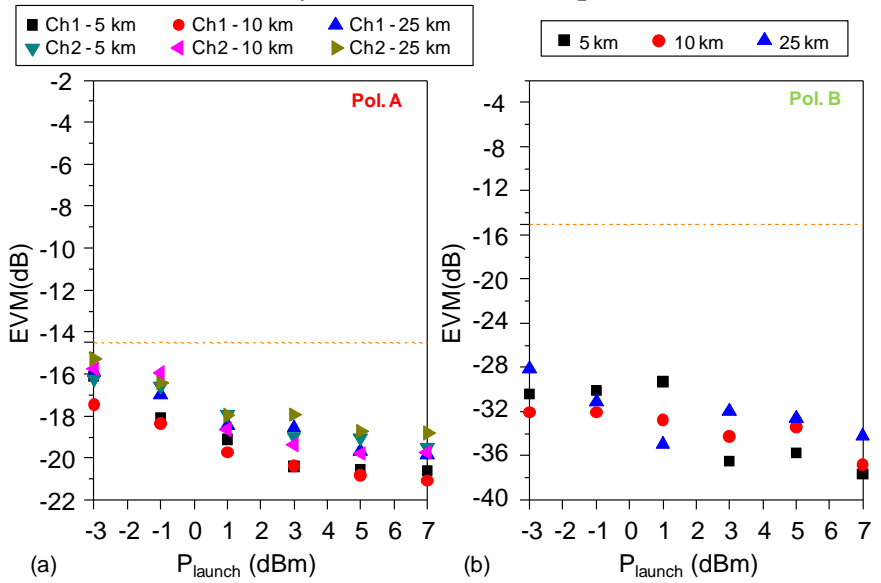

Figure 10. EVM vs. fiber length vs. optical launch power for (a) UWB Ch 1 and Ch 2 in Pol. A, and (b) WiMAX (10 MHz BW) in Pol. B.

\section{B. Adjacent-channel polarization multiplexing}

In this case, the adjacent-channel polarization multiplexing configuration already described in Figure 7(c) is analyzed. In the setup shown in Figure 8, the wireless service 1 is a UWB signal that uses sub-band \#2 and sub-band \#3 (center frequencies of $3.96 \mathrm{GHz}$ (Ch 2) and $4.448 \mathrm{GHz}$ (Ch 3), respectively) and the wireless service 2 is the WiMAX $3.5 \mathrm{GHz}$ signal described in Table I. The two orthogonal polarizations shown in Figure 7(a) are employed in this case.

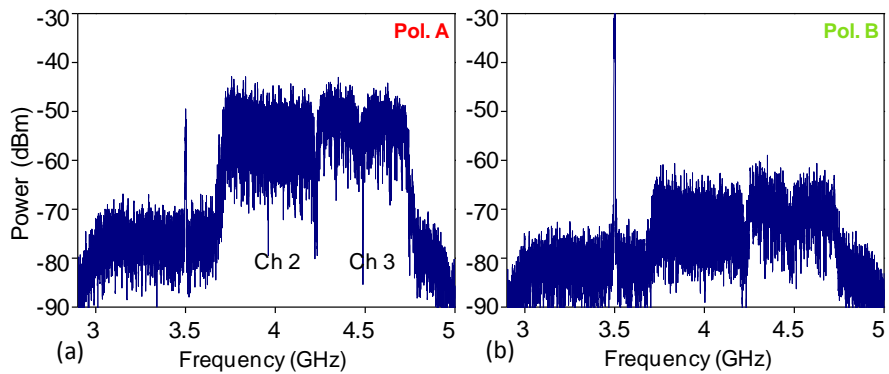

Figure 11. RF received spectrum after $25 \mathrm{~km}$ SSMF transmission measured with out-of-band coexistence in (a) point (5) Pol. A, and (b) point (6) of Figure 8 Pol. B. RBW = $1 \mathrm{MHz}$.

Figure 1 shows RF spectrum after $25 \mathrm{~km}$ fiber transmission at point (5) and (6) of the setup. Figure 11(a) shows the RF spectrum of the $400 \mathrm{Mbit} / \mathrm{s}$ MB-OFDM UWB transmission using Pol. A. It should be noticed that orthogonal polarizationmultiplexed WiMAX signal appears as a cross-polarization residual crosstalk. Figure 11(b) is the $10 \mathrm{MHz}$ bandwidth WiMAX signal in Pol. B. In this case, rejection ratio between under-study signal and cross-polarization residual crosstalk is about $30 \mathrm{~dB}$.

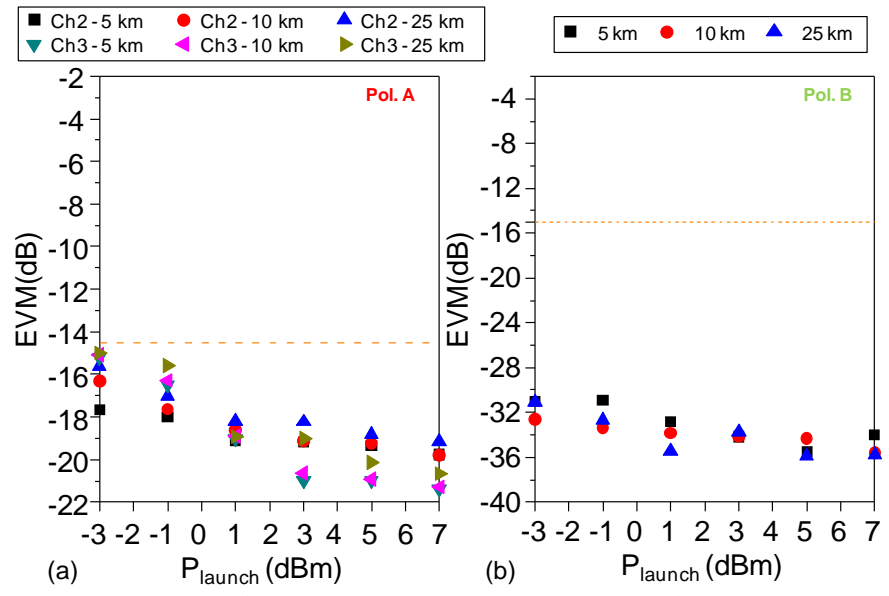

Figure 12. EVM vs. fiber length vs. optical launch power for (a) UWB Ch 2 and Ch 3 in Pol. A, and (b) WiMAX (10 MHz BW) in Pol. B.

Figure 12 shows the EVM measured for each wireless service for the adjacent-channel polarization multiplexing configuration. UWB (Pol. A) results are presented in Figure 12(a) indicating that PON transmission at $25 \mathrm{~km}$ distance is feasible in all cases under study. However, for low optical power launch values, the EVM is close to $-14.5 \mathrm{~dB}$ resulting in a poor transmission. On the other hand, WIMAX EVM results presented at Figure 12(b) are always under the $-15 \mathrm{~dB}$ EVM threshold in WiMAX, demonstrating the successful transmission.

In Figure 13, the effect of different WiMAX BW on WiMAX EVM results is analyzed. Figure 13(a) shows the cochannel configuration, and Figure 13(b) the adjacent channel configuration WiMAX EVM measurements. These results confirm that adjacent-channel polarization multiplexing outperforms the co-channel polarization multiplexing scheme. this improvement is close to $2 \mathrm{~dB}$ EVM for each WiMAX 
BW. Otherwise, it should be noticed that EVM decreases with a larger WiMAX bandwidth. For example, the EVM value for WiMAX $5 \mathrm{MHz}$ BW at $-1 \mathrm{dBm}$ optical power is close to $-36 \mathrm{~dB}$ EVM, whereas for WiMAX $20 \mathrm{MHz} \mathrm{BW}$ at $-1 \mathrm{dBm}$ optical power is $-29 \mathrm{~dB}$ EVM.
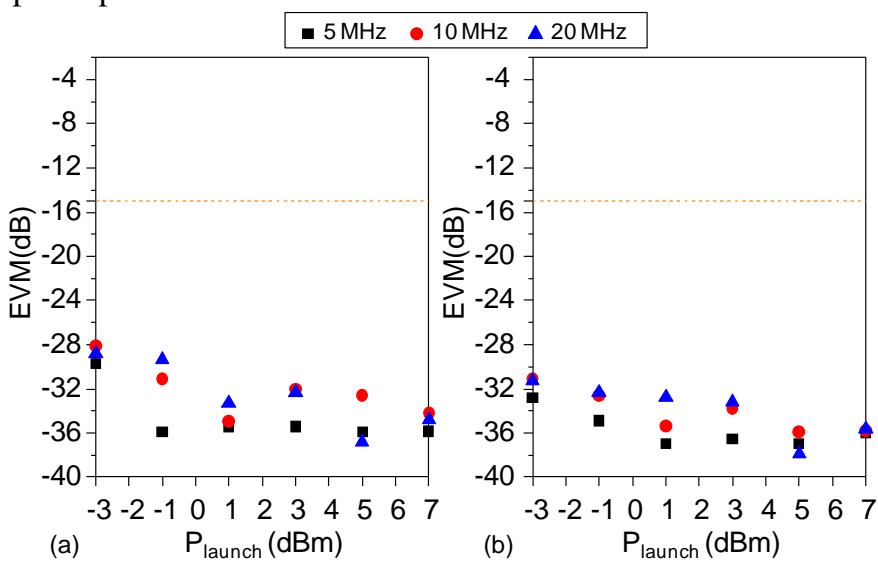

Figure 13. WiMAX EVM vs. WiMAX BW vs. optical launch power at $25 \mathrm{~km}$ for (a) co-channel, and (b) adjacent-channel interferer case.

The study of the co-channel and adjacent-channel polarization multiplexing configurations indicates that PON distances of $25 \mathrm{~km}$ fiber transmission are feasible and that EVM values depend on optical launch power at fiber in all the cases. However, the adjacent-channel configuration outperforms co-channel configuration in terms of EVM. This is due to the residual polarization crosstalk, which appears in-band in the co-channel configuration. The same polarization crosstalk appears out-of-band in the adjacent-channel configuration, giving better performance. Comparing the EVM results with the single UWB distribution scheme presented at Figure 4, the co-channel and the adjacent-channel PM configurations introduce an EVM penalty lower than $2.5 \mathrm{~dB}$ and $1.5 \mathrm{~dB}$, respectively.

\section{UWB polarization multiplexing}

A dual-polarization multiplex with two UWB channels, of $528 \mathrm{MHz}$ bandwidth each, is implemented and analyzed in this section. In this case, both wireless services 1 and 2, in Figure 8, are generated by two full standard ECMA-368 OFDM-UWB signals, each comprising two channels using sub-band \#2 and sub-band \#3 (center frequencies of $3.96 \mathrm{GHz}$ (Ch 2) and $4.488 \mathrm{GHz}(\mathrm{Ch} \mathrm{3)} \mathrm{respectively)} \mathrm{at} 200 \mathrm{Mbit} / \mathrm{s}$ per channel. Both services are modulated on the orthogonal polarization of this single wavelength signal as depicted in Figure 8.

Figure 14 shows the EVM performance when transmitting two MB-OFDM UWB channels using the same setup and the same fiber lengths but with only one polarization (no PM). In this case, the spectral efficiency is $0.3788 \mathrm{bit} / \mathrm{s} / \mathrm{Hz}$.

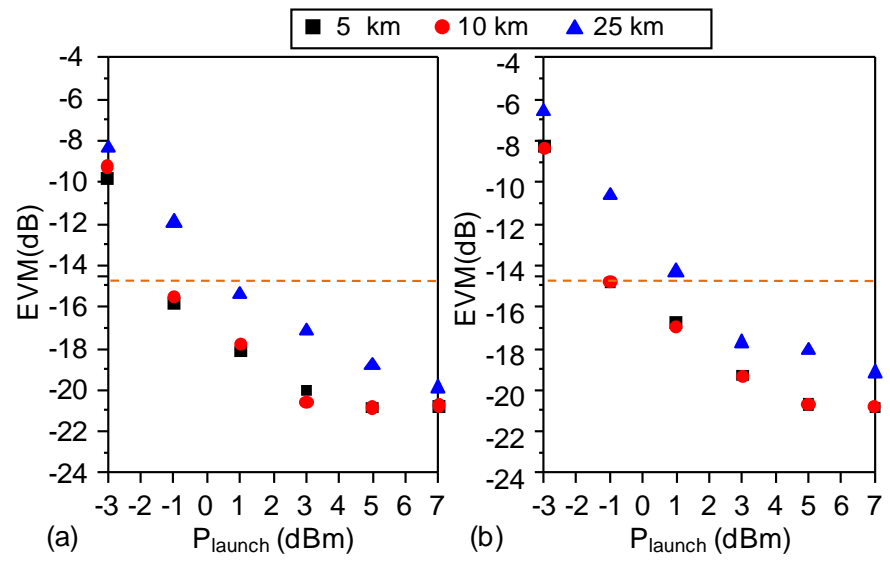

Figure 14. EVM vs. fiber length vs. optical launch power when only one polarization is used for UWB (a) channel 2 and (b) channel 3.

Figure 15 shows RF spectrum after $25 \mathrm{~km}$ SSMF transmission at point (5) and (6) of the set-up depicted in Figure 8. Figure 15(a), or top of the figure, shows spectrums for UWB joint $\mathrm{Ch} 2$ and $\mathrm{Ch} 3$ Pol. B distribution configuration, whereas Figure 15(b) or bottom of the Figure 15 corresponds to UWB Ch 2 and Ch 3 Pol. A distribution configuration. The cross-polarization residual crosstalk on the setup is around $30 \mathrm{~dB}$ for UWB Pol. B configuration distribution and $33 \mathrm{~dB}$ for UWB Pol. A configuration, as depicted on Figure 15.
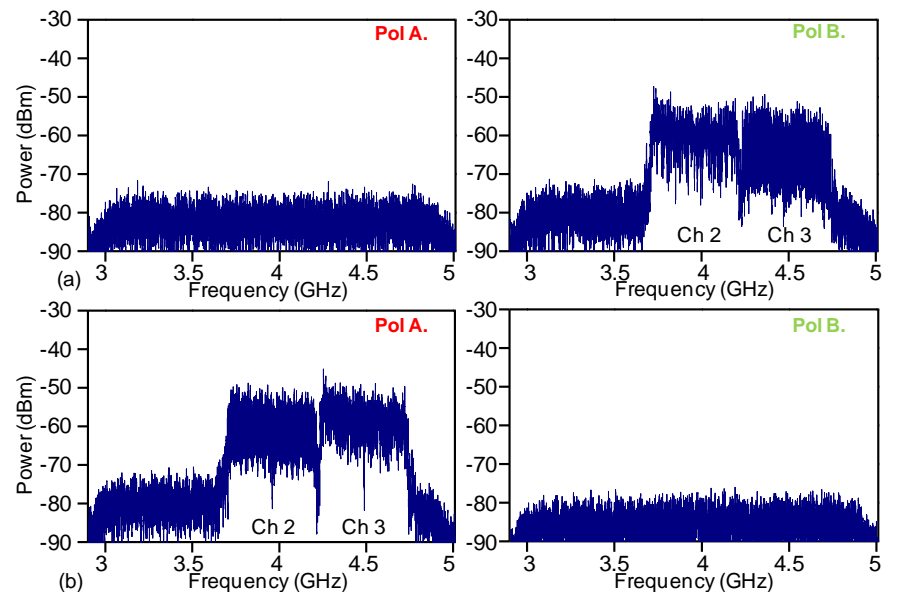

Figure 15. (a) RF received spectrum after $25 \mathrm{~km}$ SSMF transmission measured in points (5) and (6) of Figure 8, and (b) cross-polarization residual crosstalk, for Pol. A and Pol. B (top/bottom respectively). RBW $=1 \mathrm{MHz}$.

Figure 16 shows EVM measurements for the received UWB signals using polarization diversity at points (5) and (6) in Figure 8 , achieving $0.7576 \mathrm{bit} / \mathrm{s} / \mathrm{Hz}$ spectral efficiency. These results show the combined EVM for PM-UWB Ch 2 and Ch 3 after the SSMF links. EVM results indicate that both PMUWB polarizations achieve the successful communication threshold of $-14.5 \mathrm{~dB}$ EVM defined in ECMA-368 for distribution distances between 5 and $25 \mathrm{~km}$ for an optical launch power over $2 \mathrm{dBm}$ at point (3) of Figure 8. 


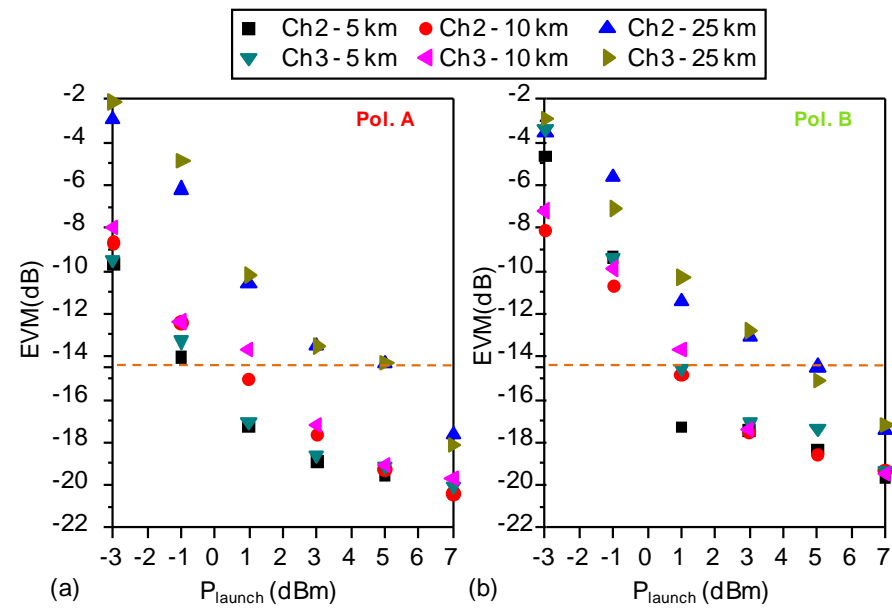

Figure 16. EVM vs. fiber length launch power vs. optical launch power for joint PM-UWB Ch 2 and Ch 3 transmission for (a) Pol. A and (b) Pol. B.

Comparing the UWB EVM results using single polarization (Figure 14), with the polarization-multiplexed approach (Figure 16), it can be observed that the interference between orthogonal polarizations induces a maximum of $2 \mathrm{~dB}$ EVM penalty. The PM-UWB configuration doubles spectral efficiency, from $0.3788 \mathrm{bit} / \mathrm{s} / \mathrm{Hz}$ to $0.7576 \mathrm{bit} / \mathrm{s} / \mathrm{Hz}$ with a penalty of $2 \mathrm{dBm}$ EVM.

\section{CONCLUSIONS}

In this paper, the feasibility of polarization-division schemes for the joint distribution of UWB and WiMAX radio has been evaluated considering four single- and orthogonal-polarization different schemes for the optical transmission co-existence.

First, a single-polarization UWB transmission with $0.3788 \mathrm{bit} / \mathrm{s} / \mathrm{Hz}$ spectral efficiency is reported achieving $25 \mathrm{~km}$ reach with $-21.5 \mathrm{~dB}$ EVM, which indicates the baseline performance. Second, the proposed co-channel polarization multiplexing scheme for UWB and WiMAX is measured. This scheme achieves $25 \mathrm{~km}$ reach with $-17.5 \mathrm{~dB}$ EVM for UWB signal and $-31 \mathrm{~dB}$ EVM for WiMAX signal. The UWB spectral efficiency obtained is $0.3788 \mathrm{bit} / \mathrm{s} / \mathrm{MHz}$. Third, the adjacent-channel UWB and WiMAX polarization multiplexing proposed scheme is demonstrated with $0.3788 \mathrm{bit} / \mathrm{s} / \mathrm{Hz}$ spectral efficiency for UWB signal transmission. Adjacent-channel polarization multiplexing scheme achieves $25 \mathrm{~km}$ reach with $-18 \mathrm{~dB}$ EVM for UWB signal and $-32.5 \mathrm{~dB}$ EVM for WiMAX signal. Finally, the UWB orthogonal polarization multiplexing scheme is reported with $0.7576 \mathrm{bit} / \mathrm{s} / \mathrm{Hz}$ spectral efficiency. This scheme achieves $25 \mathrm{~km}$ reach with $2 \mathrm{~dB}$ EVM penalty compared with UWB single-polarization distribution scheme.

The experimental results demonstrate the feasibility of the polarization-division technique proposed for typical PON distances. This technique enables the joint distribution of UWB and WiMAX. Adequate polarization tracking, photodection, filtering and amplification stages at the customer premises are required for on-the-field deployment.

\section{ACKNOWLEDGMENT}

The authors would like to thank the kind support of the RAS cluster [27], and the support of the BONE ("Building the Future Optical Network in Europe") Network of Excellence [28], both funded by the European Commission.

\section{REFERENCES}

[1] J.O. Farmer and K. Bourg, "Practical Deployment of Passive Optical Networks," IEEE Communications Magazine, pp. 136-145, June 2008.

[2] T. Koonen, M. García Larrodé, P. Urban, H. Waardt, C. Tsekrekos, J.Yang, H.Yang, H. Boom, "Fiber-based Versatile Broadband Access and In-Building Networks," IET Workshop From Access to Metro, BB Europe, December 2007.

[3] P.W. Shumate, "Fiber-to-the-Home: 1977-2007," IEEE Journal of Lightwave Technology, vol.26, no.9, pp.1093-1103, May 2008.

[4] N. Genay, P. Chanclou, F. Saliou, Q. Liu, T. Soret, L. Guillo, "Solutions for Budget Increase for the Next Generation Optical Access Network," In proceedings of ICTON '07, vol.1, pp.317-320, July 2007.

[5] L. Rerko, "Triple-Play Service Deployment: A Comprehensive Guide to Test,Measurement, and Service Assurance," JDS Uniphase Corporation, 2007.

[6] H. Pfrommer, M.A. Piqueras, V. Polo, J. Marti, "Full-duplex DOCSIS/wirelessDOCSIS Small-Scale Field Trial Employing Hybrid Fiber Radio Systems," In proceedings of the IEEE MTT-S International Microwave Symposium Digest, pp. 26-29, June 2006.

[7] A. Kim, Y. Hun Joo, Y. Kim, " $60 \mathrm{GHz}$ wireless communication systems with radio-over-fiber links for indoor wireless LANs," IEEE Transactions on Consumer Electronics, vol.50, no.2, pp. 517-520, May 2004

[8] R. Llorente, T. Alves, M. Morant, M. Beltran, J. Perez, A. Cartaxo, and J. Marti, "Ultra-Wideband Radio Signals Distribution in FTTH Networks," IEEE Photonics Technology Letters, vol.20, no.11, pp. 945947, June 2008

[9] T. Lunttila, S. Iraji, H. Berg, "Advanced coding schemes for a multiband OFDM ultrawideband system towards $1 \mathrm{Gbps}$," In proceedings of the IEEE 3rd Consumer Communications and Networking Conference, vol.1, pp. 553-557, January 2006.

[10] R. Kohno, "State of Arts in Ultra Wideband (UWB) Wireless Technology and Global Harmonization," In proceedings of the 34th European Microwave Conference, vol.2, pp. 1093-1099, October 2004.

[11] WHDI Special Interest Group URL: http://www.whdi.org/

[12] FCC 02-48, "Revision of Part 15 of the Commission's Rules regarding ultra-wideband transmission systems," April 2002.

[13] ECMA 368 International Standard, "High Rate Ultra Wideband PHY and MAC Standard," December 2005.

[14] Institute of Electrical and Electronics Engineers, Inc., IEEE Std 802.16e2005; IEEE Standard for Local and metropolitan area networks; Part 16: Air Interface for Fixed Broadband Wireless Access Systems, Amendment 2: Physical and Medium Access Control Layers for Combined Fixed and Mobile Operation in Licensed Bands and Corrigendum 1, February 2006.

[15] Westech Communications Inc., "Can WiMAX Address Your Applications?," WiMax Forum whitepaper, October 2005.

[16] T. Alves, A. Cartaxo, "Performance Degradation Due to OFDM-UWB Radio Signal Transmission Along Dispersive Single-Mode Fiber," IEEE Photonics Technology Letters, vol.21, no.3, pp. 158-160, February 2009.

[17] R. Alemany, J. Perez, R. Llorente, V. Polo, J. Marti, "Coexistence of WiMAX 802.16d and MB-OFDM UWB in radio over multi-mode fiber indoor systems," In proceedings of the International Topics Meeting on Microwave Photonics MWP'08, pp. 74-77, October 2008.

[18] Q. Zou, A. Tarighat, and A. H. Sayed, "Performance analysis of multiband OFDM UWB communications with application to range improvement," IEEE Transactions on Vehicular Technology, vol. 56, no. 6, pt. 2, pp. 3864-3878, November 2007.

[19] Institute of Electrical and Electronics Engineers, Inc., IEEE Std 802.162004; IEEE Standard for Local and metropolitan area networks; Part 16: Air Interface for Fixed Broadband Wireless Access, October 2004. 
[20] Q. Chang, J. Gao, Q. Li, Y.i Su, "Simultaneous Transmission of Pointto-Point Data and Selective Delivery of Video Services in a WDM-PON Using ASK/SCM Modulation Format," In proceedings of the Optical Fiber Communication Conference and Exposition (OFC), February 2008.

[21] H. Schmuck, "Comparison of optically millimeter-wave system concepts with regard to chromatic dispersion," Electronics Letters, vol.31, no.21, pp. 1848-1849, October 1995.

[22] Electronic Communications Committee (ECC), ECC/DEC/(07)02, "ECC Decision of 30 March 2007 on availability of frequency bands between $3400-3800 \mathrm{MHz}$ for the harmonised implementation of Broadband Wireless Access systems (BWA)".

[23] General Photonics "Reset-free polarization stabilizer - PolaStay ${ }^{\mathrm{TM}}$," http://www.generalphotonics.com/pdf/PolaStay.pdf

[24] UCELLS project URL: http://www.ict-ucells.eu

[25] DECISION No 1982/2006/EC OF THE EUROPEAN PARLIAMENT AND OF THE COUNCIL of 18 December 2006 concerning the Seventh Framework Programme of the European Community for research, technological development and demonstration activities (2007-2013).

[26] ICT - INFORMATION AND COMMUNICATION TECHNOLOGIES, A Theme for research and development under the specific programme "Cooperation" implementing the Seventh Framework Programme (2007-2013) of the European Community for research, technological development and demonstration activities, Work Programme 2007-08.

[27] Radio Access \& Spectrum Cluster organised by Unit D1 of the DG INFOSYS and MEDIA of the Commission of the European Communities URL: http://www.newcom-project.eu:8080/Plone/ras

[28] ICT-BONE NoE URL: http://www.ict-bone.eu 